\title{
BDNF promotes target innervation of Xenopus mandibular trigeminal axons in vivo Jeffrey K Huang* ${ }^{*} 1$, Karel Dorey ${ }^{\dagger 1,2}$, Shoko Ishibashi 1,2 and Enrique Amaya*1,2
}

Address: ${ }^{1}$ Wellcome Trust/Cancer Research UK Gurdon Institute, Tennis Court Road, University of Cambridge, Cambridge, CB2 1QR UK and ${ }^{2}$ The Healing Foundation Centre, Faculty of Life Sciences, University of Manchester, Oxford Road, Manchester, M13 9PT, UK

Email: Jeffrey K Huang* - jh424@cam.ac.uk; Karel Dorey - Karel.Dorey@manchester.ac.uk;

Shoko Ishibashi - Shoko.Ishibashi@manchester.ac.uk; Enrique Amaya* - Enrique.Amaya@manchester.ac.uk

* Corresponding authors †Equal contributors

Published: 3I May 2007

BMC Developmental Biology 2007, 7:59 doi:10.1186/147|-2/3X-7-59
Received: 26 September 2006

Accepted: 3 I May 2007

This article is available from: http://www.biomedcentral.com/l47I-2/3X/7/59

(c) 2007 Huang et al; licensee BioMed Central Ltd.

This is an Open Access article distributed under the terms of the Creative Commons Attribution License (http://creativecommons.org/licenses/by/2.0), which permits unrestricted use, distribution, and reproduction in any medium, provided the original work is properly cited.

\begin{abstract}
Background: Trigeminal nerves consist of ophthalmic, maxillary, and mandibular branches that project to distinct regions of the facial epidermis. In Xenopus embryos, the mandibular branch of the trigeminal nerve extends toward and innervates the cement gland in the anterior facial epithelium. The cement gland has previously been proposed to provide a short-range chemoattractive signal to promote target innervation by mandibular trigeminal axons. Brain derived neurotrophic factor, BDNF is known to stimulate axon outgrowth and branching. The goal of this study is to determine whether BDNF functions as the proposed target recognition signal in the Xenopus cement gland.
\end{abstract}

Results: We found that the cement gland is enriched in BDNF mRNA transcripts compared to the other neurotrophins NT3 and NT4 during mandibular trigeminal nerve innervation. BDNF knockdown in Xenopus embryos or specifically in cement glands resulted in the failure of mandibular trigeminal axons to arborise or grow into the cement gland. BDNF expressed ectodermal grafts, when positioned in place of the cement gland, promoted local trigeminal axon arborisation in vivo.

Conclusion: BDNF is necessary locally to promote end stage target innervation of trigeminal axons in vivo, suggesting that BDNF functions as a short-range signal that stimulates mandibular trigeminal axon arborisation and growth into the cement gland.

\section{Background}

Peripheral axon targeting comprises at least 2 morphologically distinct growth states: directional elongation, followed by terminal arborisation at the targets [1]. Target derived diffusible factors are known to control the outgrowth and branching of growing axons [2]. In Xenopus, the mandibular trigeminal nerve extends as fasciculated neurites for a visibly long distance toward the anterior facial epithelium, where in the vicinity of the cement gland, trigeminal axons turn ventrally, arborise and grow into the ventral posterior domain of the cement gland [3]. The cement gland is a transient embryonic tissue, made up of highly pigmented, mucous secreting cells. These cells transmit mechanosensory information via pressure 
sensitive receptors in trigeminal axon terminals to activate tonic inhibition response in the swimming tadpole $[4,5]$. Honore and Brivanlou have previously demonstrated that the surgical deletion of cement glands from Xenopus embryos resulted in mandibular trigeminal nerve targeting error, and proposed that a cement gland-derived chemoattractive signal operates from a short distance to control the branching and growth of mandibular trigeminal axons to the cement gland [6].

It is well known in co-culture experiments that directed outgrowth of trigeminal axons could be stimulated by tissue derived chemoattractants, termed Maxillary Factor from target maxillary/mandibular tissues [7]. It has since been demonstrated that Maxillary Factor comprises the neurotrophins BDNF and NT3 [8]. Neurotrophins are a family of secreted ligands, including NGF, BDNF, NT3 and NT4 that bind to designate Trk receptors in the nervous system to promote neuronal survival $[9,10]$. Previous studies showed that mice deficient in BDNF or NT3 displayed a profound loss of sensory neuron populations in the trigeminal ganglia and spinal cord, and die shortly after birth [9-12]. Furthermore, these mice did not exhibit any defect in the projection of trigeminal axons [8]. However, it remained unclear whether target-derived neurotrophins might function locally to promote the end stage targeting of trigeminal axons in vivo. In in vitro growth cone turning assays, growth cones from isolated spinal cord neurons could orient toward a directional gradient of neurotrophins emanating from a micropipette [13]. Moreover, regional overexpression of neurotrophins in the Xenopus central nervous system could promote localised growth and branching from neurotrophin responsive axons [14]. Additionally, neurotrophins when applied ectopically in mammalian slice cultures can promote sensory axon outgrowth from the spinal cord into the periphery [15]. Since the cement gland is a well-defined target for mandibular trigeminal axons, Xenopus might provide a useful model for the analysis of neurotrophin function in axon-target interaction in vivo. Recently it has been demonstrated that TrkB, the receptor for BDNF and NT3 is highly expressed by trigeminal and spinal sensory neurons in Xenopus embryos, which suggests that neurotrophins might play a role in peripheral sensory axon development [16]. Here we determine whether the Xenopus cement gland expresses neurotrophins, and focus on the role of BDNF during mandibular trigeminal axon target innervation in vivo.

\section{Results}

Expression of BDNF in the Xenopus cement gland during mandibular trigeminal axon development

The mandibular trigeminal nerve is a prominent cranial nerve that innervates the Xenopus cement gland (Figure 1A). During development, it exits and projects from the trigeminal ganglion as fasciculated neurites towards the cement gland. Once near the cement gland, the axons extend ventrally and then enter the ventral domain of the cement gland as branched fibers [6] (Figure 1B). To confirm the presence of local axon targeting signals in the cement gland, we repeated Honore and Brivanlou's experiment of surgically deleting the cement gland in developing embryos at St. 18, and analysed mandibular trigeminal nerve morphology at St. 29, the stage of axon target innervation by whole mount immunostaining. Consistent with their observation [6], we detected the disruption of mandibular trigeminal nerve targeting in embryos lacking the cement gland $(\mathrm{n}=10)$. Without intact cement glands, mandibular trigeminal nerves extended normally to the anterior facial epithelium, but then either stopped before the site of cement gland deletion (30\%; Figure 1C), grew away to the dorsal facial epithelium $(20 \%)$ or to the ventral epithelium $(50 \%$; Figure $1 \mathrm{D}$ and $1 \mathrm{E})$. Furthermore, most of the axons remained fasciculated and axon terminals did not arborise. This observation suggests that the cement gland is likely to provide a short range signal to promote the end stage targeting of
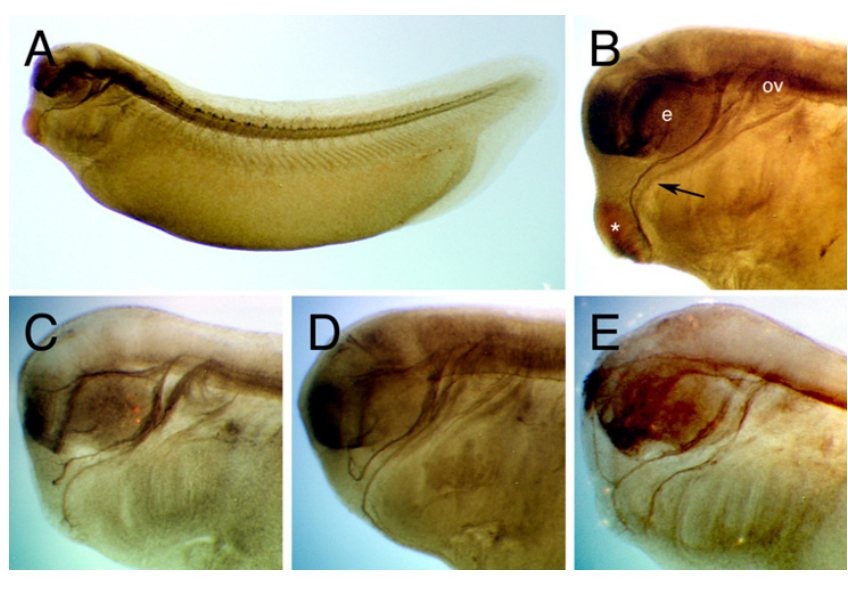

\section{Figure I}

Xenopus mandibular trigeminal nerve innervates the cement gland. (A) Whole mount immunostaining of a Xenopus embryo (St. 29) labeled with $\beta$-tubulin antibody and cleared in Murray's clearing solution reveals the embryonic axonal network, including the prominent mandibular trigeminal nerve. (B) The mandibular trigeminal nerve (arrow) extends between the eye vesicle (e) and otic vesicle (ov), and then turns ventrally before terminating at the ventral region of the cement gland (asterisk). Deletion of cement glands results in the loss of trigeminal targeting, where the axons either $(C)$ stop growth, or extended $(D)$ dorsally or $(E)$ ventrally. Because the embryos are transparent, trigeminal axons on the opposite side could also be detected. In the cement gland null embryos, trigeminal axons on either side of the face do not necessarily project erroneously in the same directions. 
mandibular trigeminal axons by stimulating branching and growth into the ventral domain of the cement gland.

Given that neurotrophins have been suggested to mediate mammalian peripheral trigeminal axon outgrowth in vitro $[8,17]$, we asked when and where neurotrophins are expressed in Xenopus embryos. We found that BDNF, NT3 and NT4 are expressed in early developing embryos (Figure 2A). Additionally, their expression increased at St. 18 during the period of primary neuronal development, and appeared to remain constant throughout all of the subsequent developmental stages examined. During development, the Xenopus mandibular trigeminal axons grow out toward the cement gland at St. 24 and terminate at the cement gland by St. 29 [6]. When we examined the expression of neurotrophins in cement glands of St. 24 embryos, we found that the cement gland is enriched in BDNF mRNA, compared to NT3 and NT4 (Figure 2A). By whole mount in situ hybridisation we detected BDNF mRNA at the neural plate in neurula stage embryos (St. 18) (Figure $2 \mathrm{~B})$, and in the posterior region of the cement gland in tailbud stage embryos at St. 24 (Figure 2C and 2E) and St. 29 (Figure 2D). The detection of BDNF mRNA in the cement gland suggests that BDNF might mediate mandibular trigeminal axon target innervation in Xenopus.

\section{Phenotype of Xenopus embryos after BDNF knockdown}

To examine BDNF function during Xenopus nerve development, we used an antisense morpholino oligonucleotide (MO) approach to knockdown BDNF expression. We identified the BDNF gene in the genome in the JGI Xenopus tropicalis genomic database [18]. We then obtained the genomic sequence of BDNF and designed a $\mathrm{MO}(\mathrm{MO}$ BDNFatg) against a 24 nucleotide sequence in the $5^{\prime}$ region of XtBDNF mRNA, including the start codon (Fig $3 \mathrm{~A}$ ). This region is $100 \%$ conserved between Xenopus laevis and Xenopus tropicalis BDNF. Furthermore, it is highly conserved in all of the BDNF genes examined in other vertebrates and mammals

First, we tested whether MO BDNFatg inhibited translation of an HA-tagged XtBDNF construct in X. laevis embryos. The HA tag was added to the C-terminus of XtBDNF (BDNF-HA), and the region surrounding the start of translation was not altered. When the BDNF-HA RNA was injected alone in $X$. laevis embryos, a specific band at $34 \mathrm{kDa}$ was detected by Western blot analysis (Figure 3B, arrowhead). However, the expression of BDNF-HA was completely inhibited by co-injection of MO BDNFatg. As control, we used an oligonucleotide designed against the $X$. tropicalis Sprouty2 gene, containing 4 mismatch nucleotides (MOC), which has previously been shown not to affect Xenopus development [19]. We found that MOC had no effect on the translational efficiency of the HA-tagged BDNF construct (Figure 3B). This

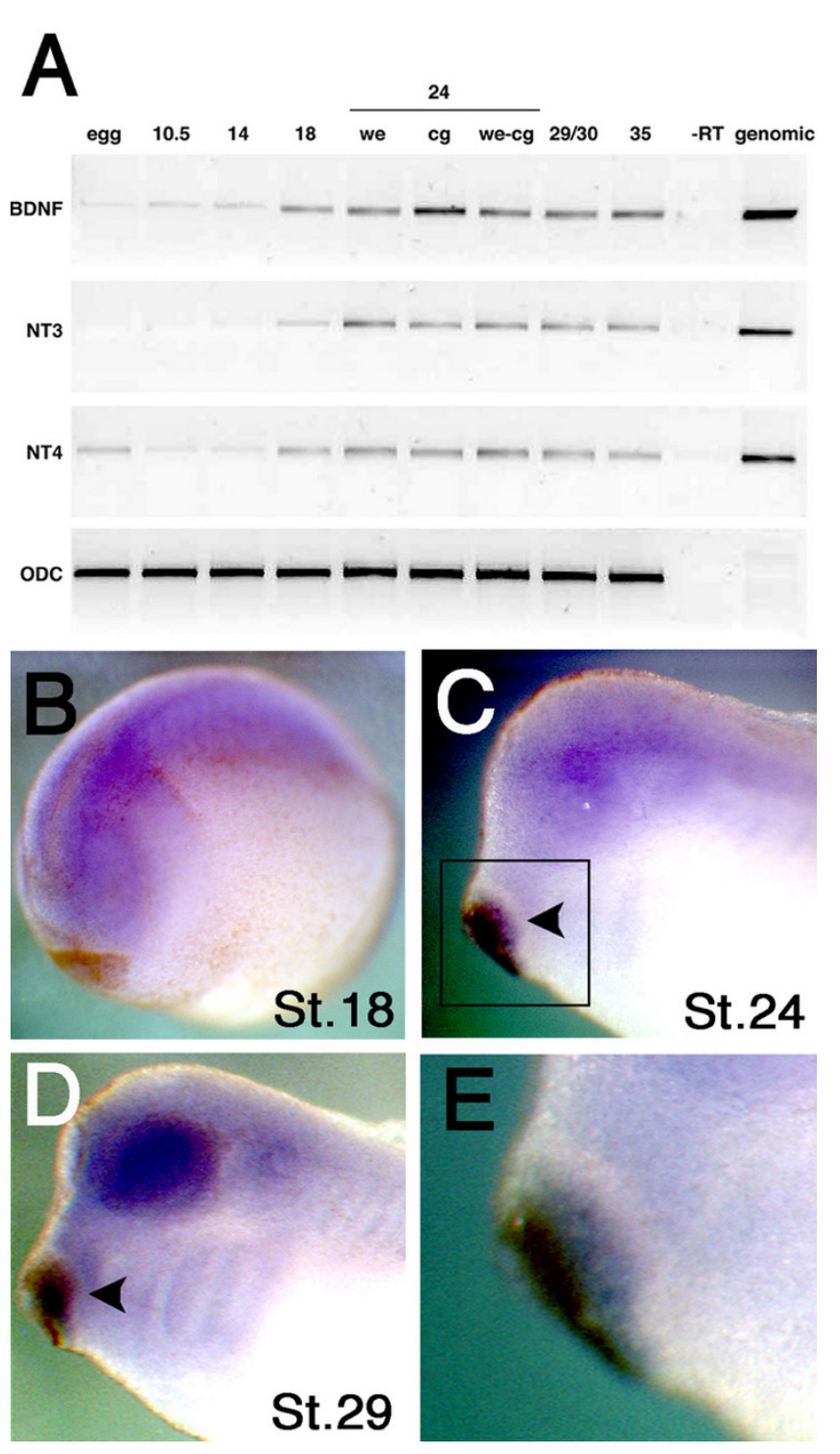

Figure 2

Detection of BDNF transcripts in the Xenopus cement gland. (A) RT-PCR shows that BDNF, NT3 and NT4 were detected throughout all stages of embryonic development. Expression of these genes increases during neurulation (St. 18). The BDNF transcript was particularly enriched in the cement gland compared to the other neurotrophin transcripts at tailbud stage (St. 24). The amount of input CDNA was confirmed with primers generated against the housekeeping gene, ornithine decarboxylase (ODC) to ensure equal loading. (B) Whole mount in situ hybridisation shows that BDNF mRNA are initially detected in the neural plate at St. 18. (C) At St. 24, $\mathrm{BDNF}$ is detected at the posterior region of the cement gland (arrowhead), which corresponds to the time of trigeminal nerve innervation. (D) At St. 29, BDNF expression remains in the cement gland and can also be detected in the eye and otic vesicles. (E) Inset from (C) shows the detection of BDNF mRNA in the cement gland at St. 24. we = whole embryo, $\mathrm{cg}=$ cement gland, we-cg = whole embryo without cement gland. 


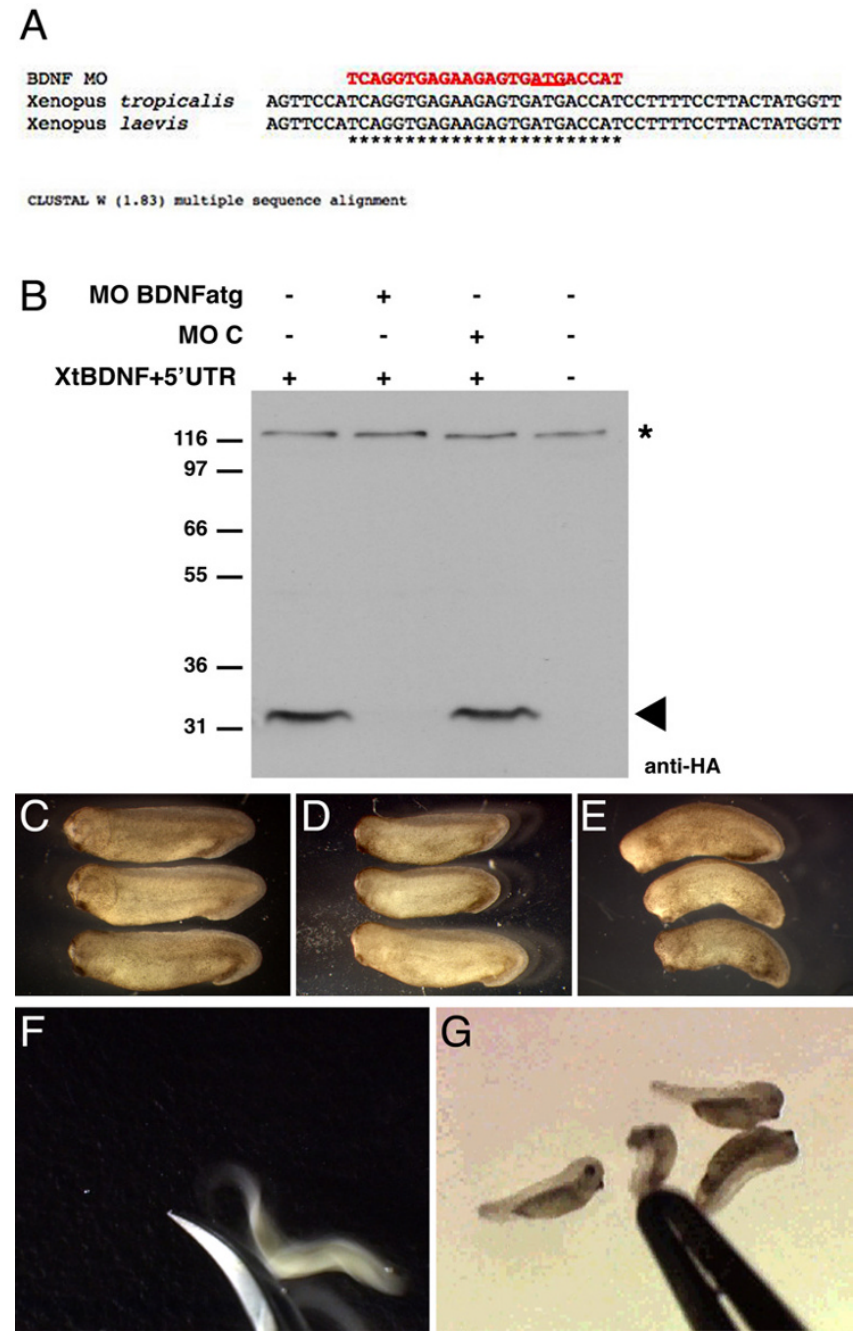

Figure 3

Effect of BDNF antisense morpholino oligonucleotides (MO) on Xenopus morphology and behaviour. (A) BDNF MO was designed against the 5' UTR and start ATG region (underlined). This region was $100 \%$ conserved between $X$. laevis and $X$. tropicalis. Asterisks indicate conserved bases. (B) Embryos were injected with $250 \mathrm{pg}$ of XtBDNF-HA mRNA alone or with $20 \mathrm{ng}$ of MO BDNFatg or the control MO (MOC). Embryos were harvested at St 12 and proteins extracts analysed by Western blotting. $X t$ BDNF was detected using anti-HA antibodies (arrowhead). The asterisk indicates a non-specific band from the same blot, which ensures equal loading in each lane. (C) Uninjected $X$. laevis embryos at St. 29. (D) Control morpholino (MOC) injected embryos at St. 29 developed normally. (E) BDNF morphants appeared slightly truncated at St. 29. (F) An uninjected tadpole with normal mechanosensory response, which escaped when probed with forceps. (G) BDNF morphants exhibited impaired mechanosensory response and did not escape when probed. showed that the BDNF MO was highly effective in knocking down BDNF translation.

To examine the effects of BDNF knockdown on axon development, we injected MOs into X. laevis, and X. tropicalis embryos. We found that all of the MO BDNFatg injected embryos $(\mathrm{n}=68 \mathrm{X}$. tropicalis, $\mathrm{n}=30 \mathrm{X}$. laevis $)$ developed without visible morphological abnormalities up to the early tailbud stage (St. 24). These animals were similar in appearance to uninjected embryos $(\mathrm{n}=56 \mathrm{X}$. tropicalis, $\mathrm{n}=50 \mathrm{X}$. laevis $)$ and MOC embryos $(\mathrm{n}=22 \mathrm{X}$. tropicalis, $\mathrm{n}=15$ X. laevis). At St. 28/29, both X. laevis and $X$. tropicalis injected with MO BDNFatg appeared slightly truncated in appearance (Figure 3E) compared to uninjected (Figure 3C) and control MOC injected embryos (Figure 3D). At St. 29, Xenopus embryos normally twitch and escape if lightly touched with an object (Figure 3F). Compared to uninjected and control MOC injected embryos which exhibit normal mechanosensory reflex (Additional file 1 and 2), we observed that the BDNF morphants appeared paralysed (Figure 3G and Additional file $3)$. Despite the lack of visible sensory reflex, the morphants continued to develop until the hatching stage (St. 32). These results show that BDNF knockdown results in consistent phenotype and behaviour in both $X$. tropicalis and $X$. laevis embryos.

\section{Analysis of mandibular trigeminal axon targeting after BDNF knockdown}

We next determined if mandibular trigeminal axon outgrowth was disrupted in MO BDNFatg injected embryos at St. 29. The X. tropicalis embryos used in our experiment were obtained from sibling mating of a recently generated transgenic line which expresses human placental alkaline phosphatase (PLAP) under the control of the neuronal specific $\beta$-tubulin promoter. The advantage in using this line is that PLAP is a heat stable and histochemically detectable enzyme [20], which enables the rapid detection of axonal projections in situ in whole embryos by a simple alkaline phosphatase (AP) reaction at any stage of development (Figure 4A and 4B). In uninjected embryos ( $\mathrm{n}=$ 23), normal projection of mandibular trigeminal axons was observed, where fasciculated axons extended to the target tissue and then turned ventrally toward the ventral domain of the cement gland (Figure 4C). In MOC embryos $(n=16)$, as well as in MO NT3 injected embryos $(\mathrm{n}=25)$, projection of mandibular trigeminal axons also appeared normal (Figure 4D and 4E). In MO BDNFatg injected embryos $(77.8 \%, \mathrm{n}=18)$, the majority of mandibular trigeminal axon appeared to project straight to the cement gland area, and did not turn toward the ventral domain of the cement gland, where they normally innervate (Figure $4 \mathrm{~F}$ ). Additionally, premature axon defasciculation was occasionally observed among the morphants $(28.6 \%)$. 

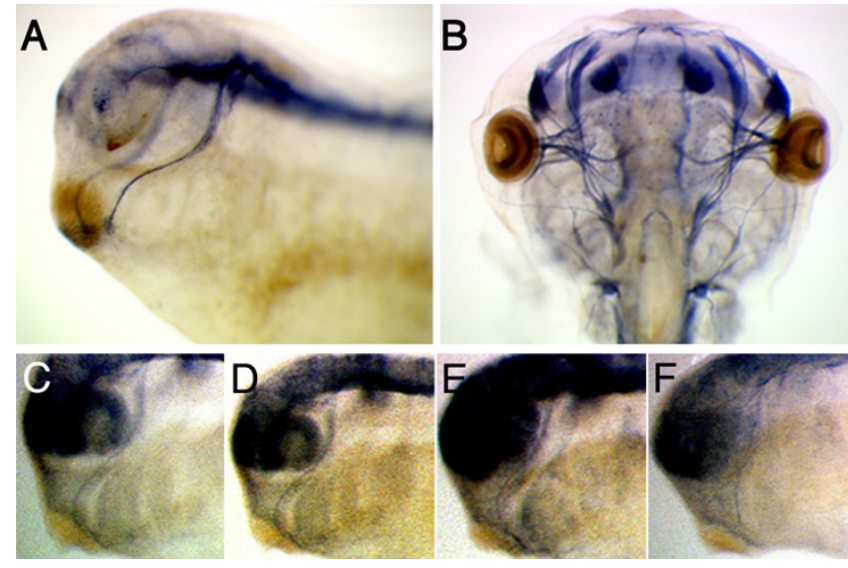

Figure 4

MO BDNFatg injected into N-PLAP transgenic $X$. tropicalis embryos exhibit possible disruption in mandibular trigeminal axon targeting. (A) A transgenic $X$. laevis embryo (St. 29) expressing N-PLAP following alkaline phosphatase reaction demonstrates the neurospecific labeling of CNS axons. The mandibular trigeminal nerve can be observed to project to the cement gland where terminal arborisation occurs. (B) The detection of cranial nerves in a transgenic tadpole (St. 47). (C) An uninjected $X$. tropicalis transgenic embryo (WT) displaying normal trigeminal nerve projection. (D) A MOC injected transgenic embryo and (E) a MO NT3 injected embryo show normal trigeminal nerve projection. (F) A MO BDNFatg injected transgenic embryo shows that the trigeminal axons appeared thinner and possibly less fasciculated. Some of the axons did not extend to the posterior cement gland where they normally innervate.

It was however difficult to assess the morphology of trigeminal axon terminals at the cement gland by AP reaction. To examine in detail whether trigeminal axons terminated into cement glands of BDNF morphants, we performed whole mount immunostaining by using antibody against acetylated $\alpha$-tubulin on $\mathrm{MO}$ injected embryos. At St. 29 the embryos were analysed by scanning confocal microscopy. In uninjected ( $\mathrm{n}=14$; Figure 5A, B and $5 \mathrm{C}$ ) and MOC injected embryos, we observed that the trigeminal axon terminals arborised and entered the ventral cement gland $(\mathrm{n}=13$; Figure 5D, E and 5F; Table 1). However, in MO BDNFatg injected embryos $(n=25)$, we observed that most trigeminal axon terminals remained fasciculated and did not arborise or grow into the cement gland (Figure 5G, H and 5I). Axonal arborisation was only detected in $16 \%$ of the morphants. This observation demonstrates that BDNF expression is necessary for trigeminal axon target innervation at the cement gland. Furthermore, it suggests that terminal arborisation might be necessary for trigeminal axon growth into the cement gland.

However, because BDNF is also crucial for the survival of sensory neuron populations [21], it remained possible
Table I: Analysis of mandibular trigeminal axon arborisation after in vivo cement gland swaps.

\begin{tabular}{lccc}
\hline & $\mathbf{n}=$ & $\begin{array}{c}\text { No } \\
\text { Arborisation }\end{array}$ & Arborisation \\
\hline WT & 14 & $0 \%$ & $100 \%$ \\
MOC & 13 & $15 \%$ & $85 \%$ \\
MO BDNFatg & 25 & $84 \%$ & $16 \%$ \\
MOC CG/WT Emb & 7 & $28 \%$ & $72 \%$ \\
WT CG/MOC Emb & 6 & $17 \%$ & $83 \%$ \\
MO BDNFatg CG/WT & 23 & $87 \%$ & $13 \%$ \\
Emb & & & \\
WT CG/MO BDNFatg & 14 & $21 \%$ & $79 \%$ \\
Emb & & &
\end{tabular}

$\mathrm{CG}=$ cement gland, $\mathrm{Emb}=$ embryo, $\mathrm{WT}=$ uninjected

that the disruption of mandibular trigeminal axon target innervation in the morphants was due to an increase in overall cell death throughout the morphants rather than directly from the loss of BDNF expression in the cement gland. To address this issue, we performed a whole mount TUNEL staining to assess cell death activity in BDNF morphants. We found that the number of cells undergoing cell death in the head region of BDNF morphants $(n=10)$ was comparable to those observed in uninjected embryos $(\mathrm{n}=$ 10) during the period of mandibular trigeminal axon targeting into the cement gland (St. 27) (Figure 6A, B and
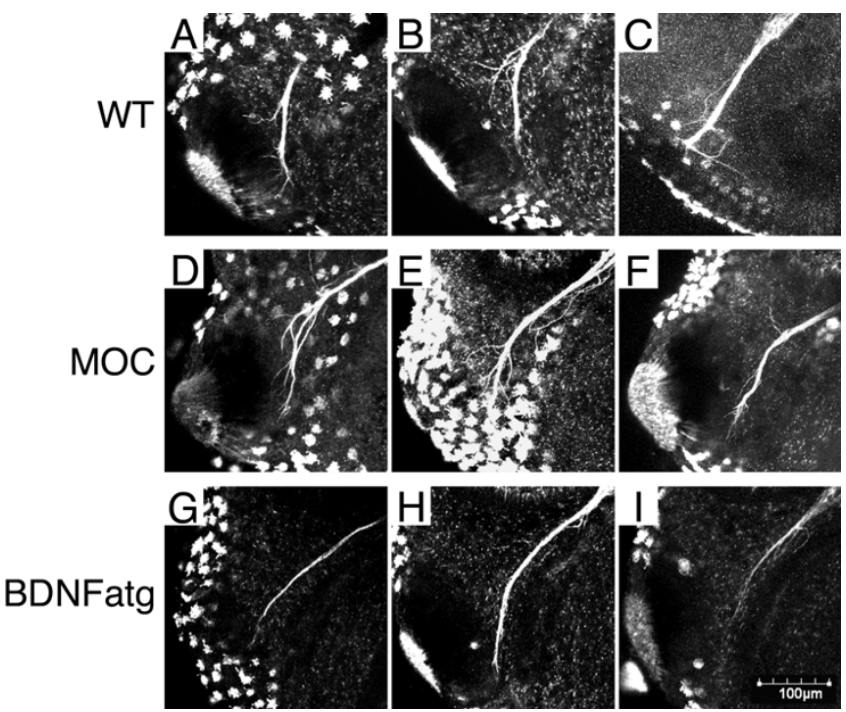

\section{Figure 5}

Decreased axon terminal arborisation in cement glands of BDNF morphants. Whole mount immunofluorescent staining of St. 29 X. laevis embryos with antiacetylated $\alpha$-tubulin shows trigeminal axon arborisation and growth into the cement gland in (A), (B) and (C) uninjected, and $(D),(E)$ and $(F)$ MOC injected embryos. $(G),(H)$ and $(I)$ In MO BDNFatg injected embryos trigeminal axons extended to the cement gland but did not arborise or grow into the cement gland. 
6E). At hatching stage (St. 32), we detected at least a 3 fold increase in cell death activity of BDNF morphants $(n=10)$ compared to uninjected embryos $(\mathrm{n}=10)$, which is 12 hours after mandibular trigeminal axons should have terminated into the cement gland (Figure 6C, D and 6E). The disruption of mandibular trigeminal axon targeting in BDNF morphants was therefore likely due to the loss of BDNF signalling and independent from cell death activities.

\section{Local BDNF expression promotes mandibular trigeminal axon arborisation in vivo}

We next determined whether mandibular trigeminal axon target innervation could be promoted by local BDNF expression by using an "in vivo cement gland substitution assay" (Figure 7A) [6]. Experimental deletion of the cement gland causes the failure of mandibular trigeminal nerve targeting (Figure 1C, D and 1E). A previous study showed that target innervation can be "rescued" by substituting the cement gland with an ectodermal animal cap explant expressing follistatin [6]. We asked if ectodermal animal cap grafts expressing BDNF when positioned in place of cement glands, could prevent trigeminal axon targeting error by stimulating local axon arborisation and growth into the grafts. In vitro transcribed mRNAs expressing either green fluorescent protein (GFP), or BDNF and GFP, were first injected into both blastomeres of Xenopus embryos at the 2-cell stage. At blastula stage (St. 8), ectodermal animal cap explants were excised and grafted onto a set of uninjected embryos at the late neurula stage (St. 18/19), which had their cement glands removed. At this stage, trigeminal ganglia have not projected their axons. The grafted embryos were then allowed to heal and develop until the late tailbud stage (St. 29). Embryos that contained intact grafts and GFP reporter expression (Figure 7B) were further analysed for trigeminal axon targeting by whole mount immunostaining with an antibody against $\beta$-tubulin. We found that the GFP expressed grafts did not prevent trigeminal axon targeting error in Xenopus embryos, as the axons either stopped short of the target $(22 \%)$, extended dorsally toward the eye $(33 \%)$, or ventrally toward the heart $(45 \%)(\mathrm{n}=9$; Figure $7 \mathrm{C}$ and Additional file 4). Furthermore, none of the grafts promoted visible axon terminal arborisation or growth into the graft. This observation was similar to that observed when cement glands were experimentally deleted (Figure 1C, D and $1 \mathrm{E}$ and $[6]$ ). Moreover, this result also demonstrates that secreted factors that might normally be expressed by animal cap cells do not influence or have a direct effect on trigeminal axon targeting. We also tested the possibility for NT3 expressed grafts to promote trigeminal axon targeting $(\mathrm{n}=11)$ by performing the same assay, but found that NT3 was unable to significantly stimulate axon arborisation or growth into the graft (Figure 7D and Additional file 4). However, when we examined embryos con-
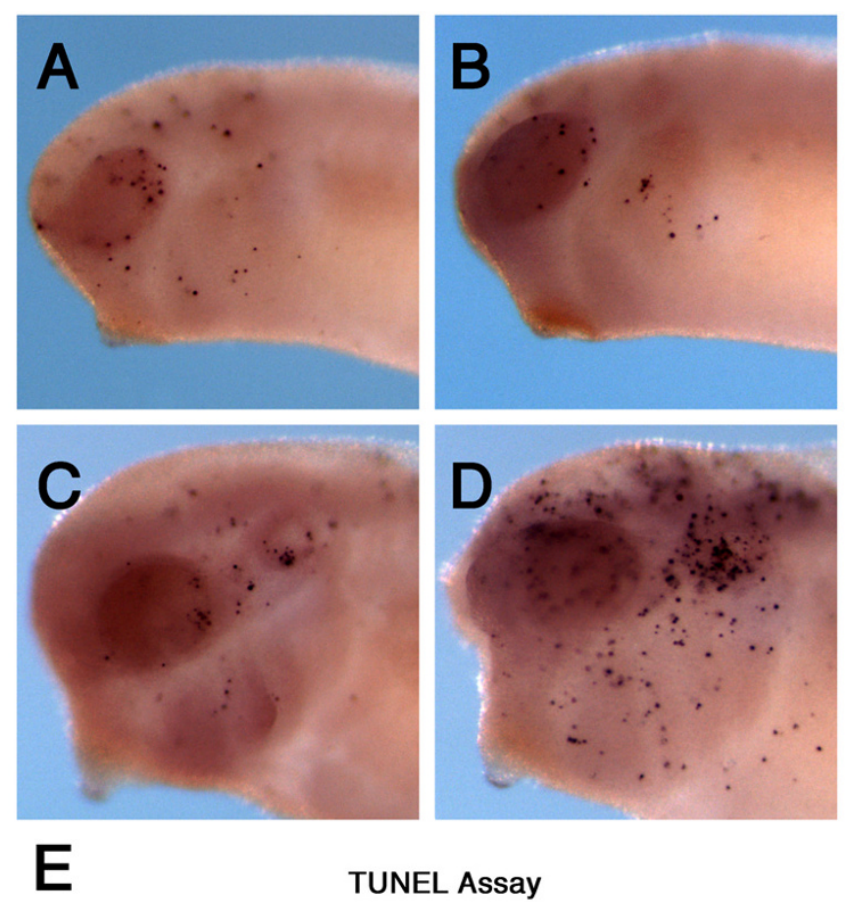

TUNEL Assay

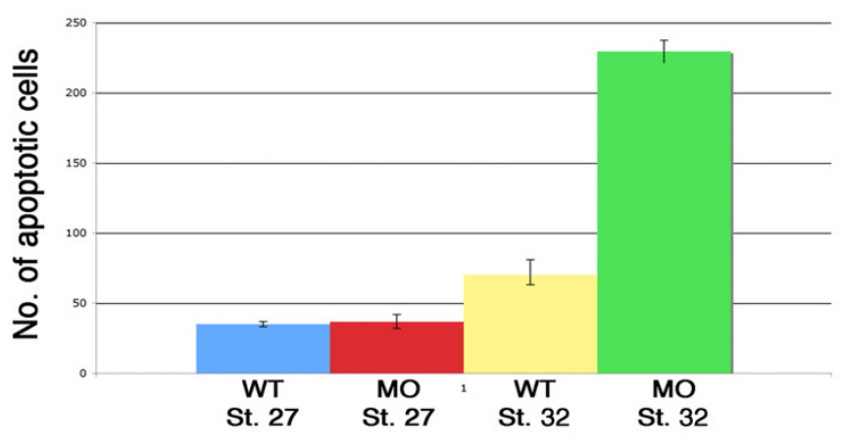

\section{Figure 6}

Increased apoptosis observed in the head region of BDNF morphants. Whole mount TUNEL staining of uninjected embryos at (A) St. 27 and (B) 32. In MO BDNFatg injected morphants, cell death activity in the head region was comparable to those in uninjected embryos at (C) St. 27, but number of cells undergoing apoptosis increased significantly at (D) St. 32. (E) Quantitation of cells undergoing apoptosis shows a 3-fold increase of cell death activity in St. 32 BDNF morphants.

taining BDNF expressed grafts, we found that the grafts promoted axon arborisation at the grafts in $82 \%$ of the embryos ( $\mathrm{n}=17$; Figure 7E and Additional file 4). This suggests that peripheral expression of BDNF could stimulate axon arborisation towards the site of its expression. Furthermore, this observation demonstrates that BDNF acts locally on trigeminal axons because the axons 


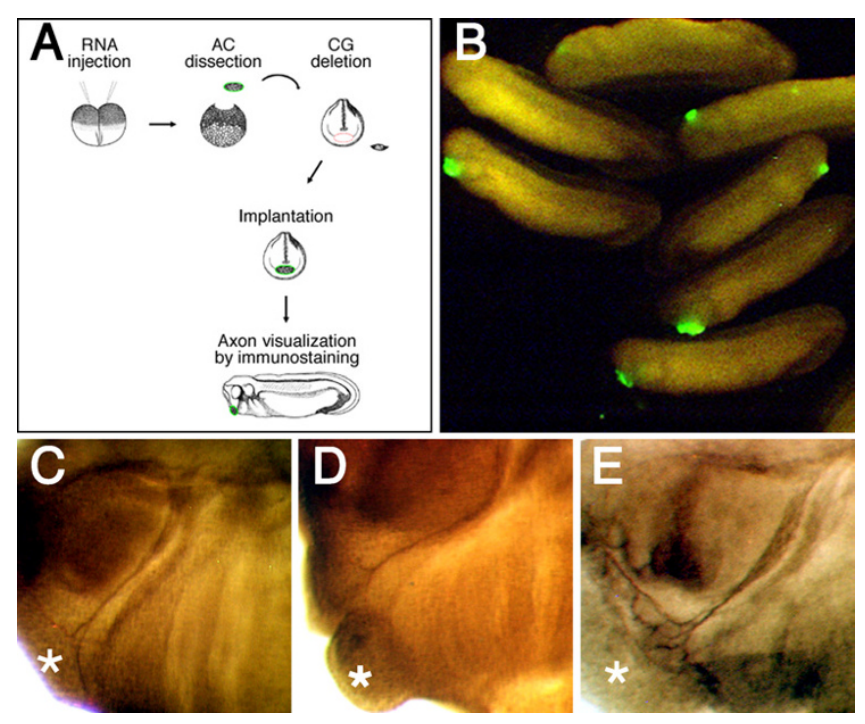

Figure 7

BDNF expressed ectodermal animal cap grafts stimulate mandibular trigeminal axon arborisation. (A) Diagram of the in vivo cement gland substitution assay. (B) Successfully grafted embryos were identified by GFP expression. (C) Neither $\mathrm{GFP}^{+}$nor (D) $\mathrm{NT3}^{+}$grafts stimulated trigeminal axon arborisation or growth onto the grafts. $(E)$ $\mathrm{BDNF}^{+}$graft stimulated axon terminal arborisation onto the graft. $A C=$ animal cap, $C G=$ cement gland, Asterisk = graft.

remained fasciculated during their projection, and target induced branching was only observed at the grafts.

While the previous experiment showed that BDNF is sufficient to stimulate terminal arborisation, we wanted to know whether BDNF expression within the cement gland in vivo is necessary for target recognition by trigeminal axon terminals. To address this, we performed an "in vivo cement gland swap" experiment by swapping cement glands between uninjected embryos and morpholino injected embryos at St.18/19 and then analysed mandibular trigeminal axon targeting at St. 29 by scanning confocal microscopy. First, we examined cement gland swaps between uninjected and MOC injected embryos. We found that MOC injected cement glands grafted onto wildtype embryos did not perturb mandibular trigeminal axon targeting, as the axons were able to arborise and grow into the cement gland grafts $(72 \%$ arborisation, $\mathrm{n}=$ 7; Figure $8 \mathrm{~A}$ and Table 1). A similar observation was also made when wildtype cement glands were grafted onto MOC injected embryos ( $83 \%$ arborisation, $n=6$; Figure $8 \mathrm{~B}$ and Table 1). We next examined cement gland swaps between uninjected and MO BDNFatg injected embryos. We found that BDNF MO injected cement glands, when grafted onto wildtype embryos, did not promote significant axon arborisation or growth into the cement glands (13\% arborisation, $\mathrm{n}=23$; Figure $8 \mathrm{C}$ and Table 1$)$. This observation was similar to that observed in MO BDNFatg injected embryos, and further demonstrates that the failure of axon arborisation at the cement gland in BDNF morphants was specific to BDNF knockdown in the cement gland and not due to a general effect elsewhere in the embryo. Furthermore, we found that wildtype cement glands, when grafted onto MO BDNFatg injected embryos, were able to rescue trigeminal axon target innervation from BDNF deficient embryos by promoting axon arborisation and growth into the grafted cement gland (79\% arborisation, $\mathrm{n}=14$; Figure $8 \mathrm{D}$ and Table 1 ). This demonstrates that trigeminal axon terminals in BDNF morphants remained responsive to target recognition signals from the cement gland. Furthermore, this suggests that BDNF expression at the cement gland is necessary for mandibular trigeminal axon target innervation.

\section{Discussion \\ Xenopus mandibular trigeminal nerve as a model for in vivo axon-target interaction studies}

Previous in vivo studies in mice determined BDNF as a trophic factor to promote sensory axon development and survival. But because BDNF null mice die soon after birth, it has not been possible to show in vivo whether BDNF functioned as a short range signal to stimulate axon target innervation [8-10]. There are several advantages in using Xenopus to study the roles of BDNF on axon targeting. The Xenopus mandibular trigeminal nerve and its target, the cement gland, provide a simple and useful in vivo system to examine the molecular basis of axon-target interaction in vertebrates. Xenopus is an excellent model for embryological studies due to the advantage of its rapid external development, and ability for micromanipulations, grafting and explant cultures. Additionally, it is amenable to genetic manipulations by germ-line transgenesis [22], as well as effective antisense morpholino oligonucleotide analysis $[23,24]$. By using these techniques, we showed that BDNF expression in the Xenopus cement gland functioned to locally promote end stage target innervation of mandibular trigeminal axons in vivo. Furthermore, because the developmental staging in Xenopus embryos is well characterised, we were able to precisely separate the effect of BDNF on target innervation from its effect on neuronal survival by analyzing distinct developmental time points.

\section{BDNF is a short-range signal to locally stimulate mandibular trigeminal axon arborisation and target innervation}

We found that BDNF knockdown did not affect the overall projection of mandibular trigeminal axons, but prevented their arborised growth into the target cement gland. Our data suggest that BDNF expressed at and near the cement gland functions to promote changes in axonal morphology necessary for trigeminal axon arborisation 


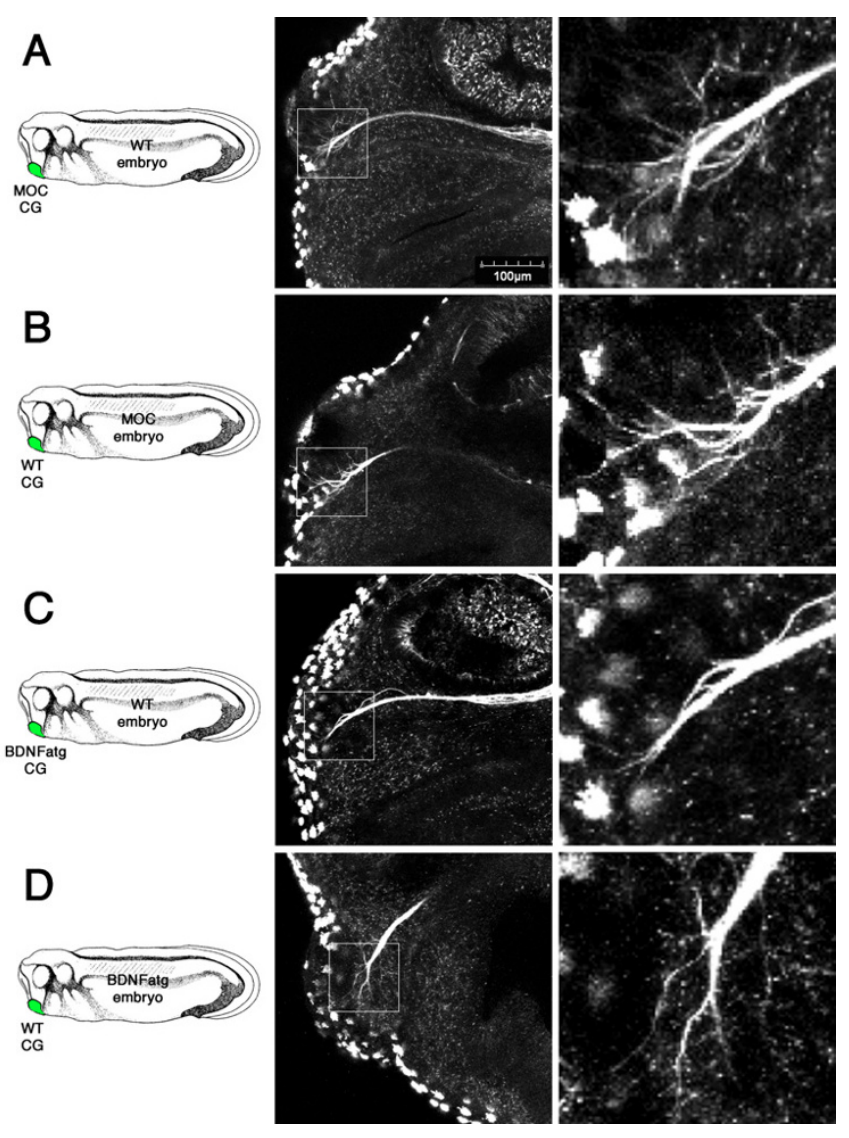

Figure 8

Wildtype cement gland grafts rescued mandibular trigeminal axon target innervation in MO BDNFatg injected embryos. In vivo cement gland swap experiments were performed between MOC injected embryos and uninjected embryos (WT). (A) MOC cement gland placed onto an uninjected embryo exhibits trigeminal axon arborisation and growth into the cement gland. (B) Similar observation was made when wildtype cement gland was placed onto a MOC injected embryo. In vivo cement gland swaps were also performed between BDNF morphants and uninjected embryos. (C) MO BDNFatg cement gland placed onto an uninjected embryo shows trigeminal arborisation at the cement gland was barely detectable. (D) Wildtype cement gland placed onto a MO BDNFatg injected embryo shows that trigeminal axons were able to arborise and enter the cement gland.

and growth into the cement gland. In the absence of BDNF, trigeminal neurons fail to arborise and enter the cement gland. By performing cement gland swapping experiments between control and experimental knockdown embryos, we were able to show that BDNF expression is required locally within the cement gland for terminal arborisation of the trigeminal nerves into the cement gland. Finally, transplantation of tissue overexpressing BDNF near the trigeminal nerve results in exces- sive branching and growth of the nerves into the BDNF expressing transplant, suggesting that BDNF is an important component of the local trigeminal nerve targeting signal, proposed by Honore and Brivanlou [6]. This finding is consistent with the findings of Nosrat and colleagues [25] and Albers and colleagues [26], who have shown that ectopic overexpression of BDNF in mice can lead to aberrant branching and sprouting of axons at the mammalian tongue.

In Xenopus embryos, trigeminal and spinal sensory neurons express TrkB during development [16]. BDNF is known to bind to the receptor tyrosine kinase TrkB, which activates several downstream signalling cascades, including the Ras-Mek-Erk and the PI3K/AKT pathways [27,28]. Activation of TrkB signalling might trigger local changes in actin cytoskeleton polymerisation within growth cones to promote arborisation of trigeminal axons [29]. BDNF can also bind to the low-affinity neurotrophin receptor p75NTR [30,31]. p75NTR plays a very important role in sensory neuron survival, outgrowth and innervation $[32,33]$, and can function in conjunction with or independently of Trk receptors [31,34]. It is thought that the specificity of TrkB activation by BDNF increases in the presence of p75NTR [30]. It is possible that at the cement gland, BDNF binds to both TrkB and p75NTR on the growth cone to promote mandibular trigeminal axon innervation and survival.

\section{Possible involvement of other neurotrophins during mandibular trigeminal axon outgrowth}

We detected the expression of other neurotrophins NT3 and NT4 in the cement gland, although their expression level was not enriched there, compared to BDNF. In mammalian in vitro axon outgrowth assays, NT3 was suggested as the dominant neurotrophin that stimulates axon outgrowth [8]. It remains possible that NT3 and NT4 might also play a role in mandibular trigeminal axon development in Xenopus. However we tested the effect of NT3 on mandibular trigeminal axon targeting by using the in vivo cement gland graft experiment, and found that NT3 did not appear to stimulate visible arborisation or target innervation. We also tested NT3 MO in Xenopus, and found that it did not have a major effect on trigeminal axon targeting at the stages we examined (St.29), and the embryos were not paralysed (See Additional file 5). In contrast, mandibular trigeminal axons rarely arborised or entered cement glands where BDNF expression was knocked down, therefore BDNF appears to be the dominant local signal that promotes local axon arborisation at the cement gland. We did, however, observe occasional arborisation at the cement gland in some BDNF morphants $(16 \%)$. It is possible that the occasional arborisation was caused by other signals, or by low level BDNF expression from incomplete BDNF knockdown at the 
cement gland. The nerve growth factor, NGF, is known to stimulate axon outgrowth from avian and mammalian trigeminal neurons in culture $[35,36]$. However, NGF is not likely to stimulate mandibular trigeminal axon innervation in Xenopus embryos, as neurons isolated during the stages when trigeminal nerves innervate the cement gland are not responsive to NGF $[13,37]$, and do not express the NGF receptor TrkA [38]. Nevertheless we cannot exclude a role for NGF in later stage maintenance of trigeminal neurons, as NGF is known to be expressed in the nervous system in later stage tadpoles [39]. Another neurotrophin family member, glial derived neurotrophic factor, GDNF has also been suggested to play a role in the outgrowth and maintenance of peripheral trigeminal axons [40,41]. Recently, GDNF was isolated in Xenopus and shown to be expressed in the maxillary facial region of Xenopus embryos during development [42]. But it does not appear to be expressed in the cement gland. It is possible that GDNF does not regulate target innervation, but plays a role in the guidance or maintenance of mandibular trigeminal axons.

\section{BDNF mediated target innervation might be necessary for mechanosensory function}

We also found that all of the BDNF morphants appeared paralysed. This might be due to the loss of BDNF responsive neurons within the trigeminal ganglia and spinal cord, since BDNF expression is necessary for sensory neuron survival and maintenance $[9,10]$. Neurotrophins and their receptors have been suggested to play a role in the development of cutaneous mechanoreceptors [43]. Recently, BDNF expression in mouse was shown to regulate the development of slowly adapting mechanoreceptors in cutaneous sensory neurons, independently from its role in promoting cell survival [44]. It is possible that local BDNF expression might provide a signal to stimulate mechanoreceptor differentiation in Xenopus sensory axons, but this remains to be determined.

\section{Conclusion}

BDNF is expressed in the cement gland during mandibular trigeminal axon development, and is required to directly stimulate the end stage targeting of Xenopus mandibular trigeminal axons in vivo. The disruption of BDNF signalling resulted in mandibular trigeminal axon targeting failure by preventing their arborisation and growth into the cement gland. BDNF's role in end stage axon targeting appeared to be independent from its role in cell survival.

\section{Methods \\ RT-PCR}

Total RNA was extracted from embryos using TRIzol according to manufacturer's instructions (Invitrogen). Ten cement glands gave similar total RNA concentration as one whole embryo. After reverse transcription using Superscript II (Invitrogen), cDNAs were subjected to PCR using the following primers in 5 ' to 3 ' direction: $B D N F$ forward: CAA GTA CCT TTG GAG CCA CC, BDNF reverse: CTA TCC ATG GTG AAA GCC CGC ACG; NT3 forward: ATG TGT CTA TTC TTA TCC ACC GCC, NT3 reverse: CAT CAA AGC ACC ATA CCA AAG CC; NT4 forward: CAT TGC TTT TTG TCT ACA CCT CGG, NT4 reverse: TCA TAC TGT TGT GCC ATC TGC; ODC [45]. Samples were taken after 29 cycles of amplification for BDNF, NT3 and NT4 in the linear range of amplification.

\section{Whole-mount in situ hybridisation and immunohistochemistry}

In situ hybridisation was performed following the protocol of Harland [46]. Antisense probes were generated from pX112-13 containing partial Xenopus BDNF cDNA (S. Cohen-Cory, UC Irvine). Immunostaining was performed as previous described [47]. Briefly, embryos were fixed in $4 \%$ paraformaldehyde and stored in methanol at $-20^{\circ} \mathrm{C}$ until use. The embryos were then rehydrated and washed twice with BBT (1\%BSA, $0.1 \%$ Tx-100 in $1 \times$ PBS) for 1 hour, once with BBT $+5 \%$ heat treated lamb serum for 1 hour, and then incubated overnight with mouse monoclonal antibodies against $\beta$-tubulin isotype I+II (Product No. T8535, Sigma) for peroxidase detection, or anti-acetylated $\alpha$-tubulin (Product No. T-6793, Sigma) for immunoflourescence detection. The embryos were then washed four times with BBT for 1 hour, once with $\mathrm{BBT}+5 \%$ heat treated lamb serum for 1 hour, and then incubated overnight with secondary anti-mouse antibodies conjugated to HRP (Molecular Probes) or to Alexa Fluor 568 (Molecular Probes). Embryos then were washed once with BBT for 1 hour and 3 times with PBT (0.1\%Tween in $1 \times$ PBS) for 1 hour, then with PBT overnight. Then the embryos were transferred to methanol before peroxidase detection by ImmnunoHisto Peroxidase Detection kit (Pierce), or scanning confocal microscopy analysis. For viewing, the embryos were cleared in Murray's clearing solution (2:1, Benzyl Benzoate:Benzyl alcohol).

\section{Morpholino oligonucleotides}

Antisense morpholino oligonucleotides (MOs) were purchased from Gene Tools LLC. Genomic sequence encompassing the start ATG region of $X$. tropicalis BDNF was obtained from Ensembl [48]: 5'-ATGGTCATCACTCTTCTCACCTGA-3'. As a control, a morpholino designed against the ATG of Xt Sprouty 2 containing 4 mismatch was used: 5'-GCCTTTTAGTACTCTCGTGTCCTTC-3' [19]. $X$. tropicalis embryos were injected with 5-8 ng total MOs along with Micro-ruby fluorescent dextran (Invitrogen) at the one cell stage. X. laevis embryos were injected with 20 ng total MOs together with in vitro transcribed GFP mRNA into both blastomeres at the 2 cell stage. Embryos that 
were positive for fluorescence were further analysed for trigeminal axon targeting by whole mount immunostaining.

\section{Western blot}

pCS2 5'UTR+XtBDNF was generated by PCR on genomic DNA using the following primers 5' CTG GAT CCA GAT GTT CCT AAT TCC TGT 3' and 5' GAC CAT TAA AAG GGG AAG ATA TCC ATA CGA TGT TCC AGA TTA CGC TTG AGA ATT CAG 3'. After digestion with EcoRI and BamHI, the PCR product was subcloned into pCS2 and verified by sequencing. Synthetic mRNA ( $250 \mathrm{pg})$ derived from this plasmid was injected in 1-cell stage $X$. laevis embryos alone or with $20 \mathrm{ng}$ of MO BDNFatg or MOC. Embryos were harvested at St 12 and homogenized in Lysate Buffer (150 mM NaCl, $20 \mathrm{mM}$ Tris pH7.5, $5 \mathrm{mM}$ EDTA/EGTA and Proteases Inhibitors (Roche)). The equivalent of one embryo was loaded and fractionated on an SDS-PAGE gel. After electrophoresis, proteins were transferred to PDVF membrane (Millipore) and membranes were probed with anti-HA HRP conjugated (clone 3F10, 1667475, Roche) followed by ECL (Amersham).

\section{Whole mount TUNEL staining}

Fixed embryos were washed twice with PBTw $(0.2 \%$ in 1 $\times$ PBS) and twice with $1 \times$ PBS. For end labelling, the embryos were washed with terminal deoxynucleotidyl transferase (TdT) buffer (GibcoBRL) for 30 minutes and then incubated with $0.5 \mu \mathrm{M}$ digoxigenin-UTP and $150 \mathrm{U} /$ $\mathrm{ml}$ TdT in TdT buffer overnight. The embryos were then washed twice with $1 \times$ PBS containing $1 \mathrm{mM}$ EDTA for 1 hour at $65^{\circ} \mathrm{C}$, then four times with $1 \times$ PBS for 1 hour at room temperature. For detection and chromogenic reaction, embryos were washed with PBT, blocked with PBT $+20 \%$ goat serum, and then incubated overnight with anti-digoxigenin antibody coupled to alkaline phosphatase in PBT $+20 \%$ goat serum. The embryos were then washed extensively before performing an alkaline phosphatase reaction.

\section{Xenopus transgenesis}

Human placental alkaline phosphatase (PLAP) cDNA was removed from RISAP vector (C. Cepko, Harvard) by SalI and SpeI and subcloned into XhoI and XbaI site of $\mathrm{pCS}^{+}$ plasmid to generate CS2-PLAP. CMV promoter was then replaced by the N- $\beta$-tubulin (NBT) promoter by SalI and HindIII ligation to generate N-PLAP. Generation of transgenic $X$. tropicalis embryos by restriction enzyme mediated integration (REMI) was carried out according to Kroll and Amaya [22], with the following modifications; $8 \times 10^{5}$ nuclei of a reaction was diluted in $130 \mu \mathrm{l}$ of $\mathrm{MOH}$, and injected into eggs using nuclear transplantation needles with an inner diameter between $40-60 \mu \mathrm{m}$ and a flow rate of $0.2 \mu \mathrm{l} / \mathrm{min}$; injection buffer was $0.1 \times \mathrm{MMR}$ in $6 \%$ Ficoll. In each reaction, $100 \mathrm{ng}$ of NotI linearised DNA was used. For generation of the N-PLAP line, REMI transgenesis was performed on, using linearised N-PLAP and $\gamma$ crystallin-dsRed, which was modified from? $\gamma$-crystallinGFP [49]. Embryos were grown to the larval stage (St. 47) and assessed for transgenesis by RFP expression in the lens. Those with RFP expression were grown until adulthood. For morpholino experiments, sibling matings between transgenic male and female frogs were performed, and the fertilised embryos were used for injection. We found at least $65 \%$ of the F1 embryos expressed PLAP.

\section{Whole mount alkaline phosphatase reaction}

Xenopus embryos were fixed with $4 \%$ paraformaldehyde in $1 \times$ PBS for 1 hour at room temperature, washed 3 times in $1 \times \mathrm{PBS}$, then endogenous alkaline phosphatase was inactivated by incubation at $65^{\circ} \mathrm{C}$ for up to 1 hour in $1 \times$ PBS. The embryos were then incubated in AP buffer (100 $\mathrm{mM}$ Tris, $\mathrm{pH}$ 9.5, $100 \mathrm{mM} \mathrm{NaCl}, 50 \mathrm{mM} \mathrm{MgCl} 2$ ) for 15 minutes followed by AP reaction (NBT/BCIP tablet (Roche)) for 15-30 minutes in the dark. Once staining was detected, the embryos were fixed in MEMFA for 1 hour, and transferred to methanol for storage. For whole mount imaging, embryos were bleached in $1 \% \mathrm{H}_{2} \mathrm{O}_{2}, 5 \%$ Formamide and $0.5 \times$ SSC, transferred to methanol, then viewed with Murray's clearing solution (2:1, Benzyl Benzoate:Benzyl alcohol).

\section{Microdissections}

In vivo cement gland substitution

Capped mRNAs were synthesized using the mMessage mMachine kit (Ambion) for the following cDNAs: mouse BDNF from BDNF-pGem4Z (K. Albers, U Pittsburgh) and GFP from pCXGFP3. Embryos were injected with approximately $250 \mathrm{pg}$ RNAs in the animal pole of both blastomeres at the 2 cells stage according to established protocols. Developmental staging was assessed as described (Nieuwkoop and Faber, 1967). Cement gland deletion and substitution assay were performed as described with modifications [6]. All embryos were transferred to $0.4 \times$ MMR saline solution containing $2 \%$ Ficoll for microdissection. Injected embryos at blastula stage (St. 8 ) and uninjected embryos at neurula stage (St. 18/19) were placed adjacent to one another in small chambers made in agarose-plated petri dishes to keep the embryos in place. Animal caps were dissected from blastula embryos, followed by the removal of the pigmented cement gland from neurula embryos using fine forceps. The caps were then trimmed and fitted onto the cement gland-null neurula embryos. These embryos were kept undisturbed for at least 3 hours following microsurgery to heal, and then transferred to Petri dishes containing $0.1 \times$ MMR until late tailbud stage. 
In vivo cement gland swaps

Antisense morpholino oligonucleotides (20 pg MO BDNFatg or MOC) together with 250 pg mRNA encoding GFP were injected in the animal pole of both blastomeres at the 2 cells stage in $X$. laevis according to established protocols. At the same time, uninjected embryos from the same fertilisation were raised in separate dishes containing 0.1 $\times$ MMR. Developmental staging was assessed as described (Nieuwkoop and Faber, 1967). MO injected embryos were confirmed by the detection of GFP fluorescence before the in vivo swap experiments. All embryos were transferred to $0.4 \times \mathrm{MMR}$ saline solution containing $2 \%$ Ficoll for microdissection. MO injected embryos and uninjected embryos at neurula stage (St. 18/19) were placed adjacent to one another in small chambers made in agarose-plated petri dishes to keep the embryos in place. Pigmented cement glands were dissected from both embryos using fine forceps and exchanged with each other. The embryos were then kept undisturbed for at least 3 hours following microsurgery to heal, and then transferred to Petri dishes containing 0.1 × MMR until St.29/30 before fixation and analysis.

\section{Authors' contributions}

$\mathrm{JKH}$ and KD contributed equally to this work. JKH conceived the experiments, JKH and KD designed, executed and analysed the experiments, and wrote the paper. SI generated the N-PLAP transgenic $X$. tropicalis line. EA participated in the design and analysis of the study, contributed to the writing of the paper and was the overseer of the project. All authors read and approved the final manuscript.

\section{Additional material}

\section{Additional File 1}

A movie showing uninjected $\mathrm{X}$. tropicalis embryos (St.32) exhibiting normal mechanosensory response.

Click here for file

[http://www.biomedcentral.com/content/supplementary/1471213X-7-59-S1.mov]

\section{Additional File 2}

A movie showing control morpholino injected $\mathrm{X}$. tropicalis embryos (St.32) exhibiting normal mechanosensory response.

Click here for file

[http://www.biomedcentral.com/content/supplementary/1471213X-7-59-S2.mov]

\section{Additional File 3}

A movie showing BDNF morpholino injected $\mathrm{X}$. tropicalis embryos (St.32) exhibiting paralysis.

Click here for file

[http://www.biomedcentral.com/content/supplementary/1471-

213X-7-59-S3.mov]

\section{Additional File 4}

Additional images of in vivo cement gland grafts.

Click here for file

[http://www.biomedcentral.com/content/supplementary/1471213X-7-59-S4.jpeg]

\section{Additional File 5}

A movie showing NT3 morpholino injected X. tropicalis embryos (St.32) exhibiting normal mechanosensory response.

Click here for file

[http://www.biomedcentral.com/content/supplementary/1471213X-7-59-S5.mov]

\section{Acknowledgements}

We thank C. Cepko (Harvard), K. Albers (U Pittsburg), and S. Cohen-Cory (UC Irvine) for reagents. This work was supported by the National Institutes of Health (RRI322I) and a Wellcome Trust Senior Research Fellowship to E.A.

\section{References}

I. Bhide PG, Frost DO: Stages of growth of hamster retinofugal axons: implications for developing axonal pathways with multiple targets. I Neurosci 199I, I I(2):485-504.

2. Tessier-Lavigne M, Goodman CS: The molecular biology of axon guidance. Science 1996, 274(5290): I| 23-1|33.

3. Davies SN, Kitson DL, Roberts A: The development of the peripheral trigeminal innervation in Xenopus embryos. J Embryol Exp Morphol 1982, 70:215-224.

4. Boothby KM, Roberts A: The stopping response of Xenopus laevis embryos: behaviour, development and physiology. J Comp Physiol $[A]$ 1992, I 70(2): I7|-180.

5. Roberts A, Blight AR: Anatomy, physiology and behavioural role of sensory nerve endings in the cement gland of embryonic Xenopus. Proc R Soc Lond B Biol Sci 1975, I 92( I I 06): I I I-I 27.

6. Honore $E$, Hemmati-Brivanlou $A$ : In vivo evidence for trigeminal nerve guidance by the cement gland in Xenopus. Dev Biol 1996 , I 78(2):363-374.

7. Lumsden AG, Davies AM: Chemotropic effect of specific target epithelium in the developing mammalian nervous system. Nature 1986, 323(6088):538-539.

8. O'Connor R, Tessier-Lavigne M: Identification of maxillary factor, a maxillary process-derived chemoattractant for developing trigeminal sensory axons. Neuron 1999, 24(I): 165-178.

9. Ernfors $P$, Lee KF, Jaenisch R: Mice lacking brain-derived neurotrophic factor develop with sensory deficits. Nature 1994, 368(6467): $147-150$.

10. Jones KR, Farinas I, Backus C, Reichardt LF: Targeted disruption of the BDNF gene perturbs brain and sensory neuron development but not motor neuron development. Cell 1994, 76(6):989-999.

II. Ernfors P, Lee KF, Kucera J, Jaenisch R: Lack of neurotrophin-3 leads to deficiencies in the peripheral nervous system and loss of limb proprioceptive afferents. Cell 1994, 77(4):503-5I2.

12. Farinas I, Jones KR, Backus C, Wang XY, Reichardt LF: Severe sensory and sympathetic deficits in mice lacking neurotrophin3. Nature 1994, 369(6482):658-661.

13. Ming G, Lohof AM, Zheng JQ: Acute morphogenic and chemotropic effects of neurotrophins on cultured embryonic Xenopus spinal neurons. J Neurosci 1997, I7(20):7860-787I.

14. Cohen-Cory S, Fraser SE: Effects of brain-derived neurotrophic factor on optic axon branching and remodelling in vivo. Nature 1995, 378(6553): 192-196.

15. Tucker KL, Meyer M, Barde YA: Neurotrophins are required for nerve growth during development. Nat Neurosci 200I, 4(1):29-37.

16. Islam N, Gagnon F, Moss T: Catalytic and non-catalytic forms of the neurotrophin receptor $x$ TrkB $m R N A$ are expressed in a 
pseudo-segmental manner within the early Xenopus central nervous system. Int J Dev Biol I996, 40(5):973-983.

17. Arumae U, Pirvola U, Palgi J, Kiema TR, Palm K, Moshnyakov M, Ylikoski J, Saarma M: Neurotrophins and their receptors in rat peripheral trigeminal system during maxillary nerve growth. J Cell Biol 1993, I 22(5): 1053-1065.

18. JGI X tropicalis v4.I [http://www.jgi.doe.gov/xenopus/]

19. Sivak JM, Petersen LF, Amaya E: FGF signal interpretation is directed by Sprouty and Spred proteins during mesoderm formation. Dev Cell 2005, 8(5):689-70I.

20. Fields-Berry SC, Halliday AL, Cepko CL: $\mathbf{A}$ recombinant retrovirus encoding alkaline phosphatase confirms clonal boundary assignment in lineage analysis of murine retina. Proc Natl Acad Sci U S A 1992, 89(2):693-697.

21. Huang EJ, Reichardt LF: Neurotrophins: roles in neuronal development and function. Annu Rev Neurosci 200I, 24:677-736.

22. Kroll KL, Amaya E: Transgenic Xenopus embryos from sperm nuclear transplantations reveal FGF signaling requirements during gastrulation. Development 1996, I 22(10):3173-3183.

23. Khokha MK, Chung C, Bustamante EL, Gaw LW, Trott KA, Yeh J, Lim N, Lin JC, Taverner N, Amaya E, Papalopulu N, Smith JC, Zorn AM, Harland RM, Grammer TC: Techniques and probes for the study of Xenopus tropicalis development. Dev Dyn 2002, 225(4):499-510.

24. Nutt SL, Bronchain OJ, Hartley KO, Amaya E: Comparison of morpholino based translational inhibition during the development of Xenopus laevis and Xenopus tropicalis. Genesis 200I, 30(3): $110-113$.

25. Ringstedt T, lbanez CF, Nosrat CA: Role of brain-derived neurotrophic factor in target invasion in the gustatory system. J Neurosci 1999, 19(9):3507-35I8.

26. Krimm RF, Miller KK, Kitzman PH, Davis BM, Albers KM: Epithelial overexpression of BDNF or NT4 disrupts targeting of taste neurons that innervate the anterior tongue. Dev Biol 200I, 232(2):508-52।.

27. Huang EJ, Reichardt LF: Trk receptors: roles in neuronal signal transduction. Annu Rev Biochem 2003, 72:609-642.

28. Markus A, Patel TD, Snider WD: Neurotrophic factors and axonal growth. Curr Opin Neurobiol 2002, I 2(5):523-53I.

29. Gallo G, Letourneau PC: Regulation of growth cone actin filaments by guidance cues. J Neurobiol 2004, 58(I):92-102.

30. Bibel M, Hoppe E, Barde YA: Biochemical and functional interactions between the neurotrophin receptors trk and p75NTR. Embo J 1999, 18(3):616-622.

31. Chao MV: The p75 neurotrophin receptor. I Neurobiol 1994 25(I I): | 373-I 385 .

32. Bentley CA, Lee KF: p75 is important for axon growth and schwann cell migration during development. J Neurosci 2000 20(20):7706-77I5.

33. Lee KF, Li E, Huber LJ, Landis SC, Sharpe AH, Chao MV, Jaenisch R: Targeted mutation of the gene encoding the low affinity NGF receptor $\mathrm{p75}$ leads to deficits in the peripheral sensory nervous system. Cell 1992, 69(5):737-749.

34. Roux PP, Barker PA: Neurotrophin signaling through the p75 neurotrophin receptor. Prog Neurobiol 2002, 67(3):203-233.

35. Ozdinler PH, Ulupinar E, Erzurumlu RS: Dose and age-dependent axonal responses of embryonic trigeminal neurons to localized NGF via p75NTR receptor. J Neurobiol 2005, 62(2): 189-206.

36. Scott SA, Davies AM: Age-related effects of nerve growth factor on the morphology of embryonic sensory neurons in vitro. J Comp Neurol 1993, 337(2):277-285.

37. Lohof AM, Ip NY, Poo MM: Potentiation of developing neuromuscular synapses by the neurotrophins NT-3 and BDNF. Nature 1993, 363(6427):350-353.

38. Ming G, Song H, Berninger B, Inagaki N, Tessier-Lavigne M, Poo M: Phospholipase C-gamma and phosphoinositide 3-kinase mediate cytoplasmic signaling in nerve growth cone guidance. Neuron 1999, 23(1): 139-148.

39. Jelaso AM, DeLong C: NGF and IL-I beta are co-localized in the developing nervous system of the frog, Xenopus laevis. Int J Dev Neurosci 2005, 23(7):575-586.

40. Fundin BT, Mikaels A, Westphal H, Ernfors P: A rapid and dynamic regulation of GDNF-family ligands and receptors correlate with the developmental dependency of cutaneous sensory innervation. Development 1999, I 26( I 2):2597-26I0.
4I. Lillesaar C, Eriksson C, Johansson CS, Fried K, Hildebrand C: Tooth pulp tissue promotes neurite outgrowth from rat trigeminal ganglia in vitro. J Neurocytol 1999, 28(8):663-670.

42. Kyuno J, Jones EA: GDNF expression during Xenopus development. Gene Expr Patterns 2007, 7(3):3|3-3|7.

43. Fundin BT, Silos-Santiago I, Ernfors P, Fagan AM, Aldskogius $H$ DeChiara TM, Phillips HS, Barbacid M, Yancopoulos GD, Rice FL: Differential dependency of cutaneous mechanoreceptors on neurotrophins, trk receptors, and P75 LNGFR. Dev Biol 1997 , 190(I):94-II6.

44. Carroll P, Lewin GR, Koltzenburg M, Toyka KV, Thoenen $\mathrm{H}$ : A role for BDNF in mechanosensation. Nat Neurosci 1998, I(I):42-46.

45. Heasman J, Kofron M, Wylie C: Beta-catenin signaling activity dissected in the early Xenopus embryo: a novel antisense approach. Dev Biol 2000, 222(I): 124-134.

46. Harland RM: In situ hybridization: an improved whole-mount method for Xenopus embryos. Methods Cell Biol |99|, 36:685-695.

47. Brivanlou $\mathrm{AH}$, Harland RM: Expression of an engrailed-related protein is induced in the anterior neural ectoderm of early Xenopus embryos. Development 1989, 106(3):61 I-617.

48. Ensembl [http://www.ensembl.org/Xenopus tropicalis/]

49. Offield MF, Hirsch N, Grainger RM: The development of Xenopus tropicalis transgenic lines and their use in studying lens developmental timing in living embryos. Development 2000 , I 27(9): | 789- I 797.

Publish with Biomed Central and every scientist can read your work free of charge

"BioMed Central will be the most significant development for disseminating the results of biomedical research in our lifetime. "

Sir Paul Nurse, Cancer Research UK

Your research papers will be:

- available free of charge to the entire biomedical community

- peer reviewed and published immediately upon acceptance

- cited in PubMed and archived on PubMed Central

- yours - you keep the copyright
BioMedcentral 